\title{
Rôle du PAF-acéther dans les réactions inflammatoires et allergiques
}

De nombreux arguments expérimentaux indiquent que le PAF-acéther, directement ou par l'intermédiaire de médiateurs secondaires, joue un rôle essentiel dans la maladie asthmatique ainsi que dans d'autres types de réactions allergiques et inflammatoires, immédiates et retardées.

\section{Marina Pretolani \\ Stagiaire de recherche à l'Institut Pasteur}

Boris Vargaftig Professeur à l'Institut Pasteur

\section{ADRESSE}

M. Pretolani, B. Vargaftig : unité de pharmacologie cellulaire, unité associée Institut Pasteur/Inserm, U. 285, 25, rue du Docteur- e PAF-acéther (plateletactivating factor) est un médiateur dérivé des phospholipides membranaires cellulaires. Sa structure (1-0-alkyl-2-acétyl-snglycéryl-3-phosphorylcholine) (figure 1) a été déterminée en 1979 par Benveniste et al. [1], et Demopoulos et al. [2] ; il constitue le premier exemple de phospholipide doué d'une activité biologique. Initialement décrit comme étant originaire des basophiles, sa libération par de nombreux autres types cellulaires inflammatoires a éte démontrée. En effet, le PAFacéther est libéré par les monocytes, neutrophiles, plaquettes, macrophages, éosinophiles et les cellules endothéliales [3]. Du fait de sa production par ces diverses cellules, l'implication du PAFacéther dans les réactions allergiques et inflammatoires a été suggérée. Au cours des dernières années, la détermination de sa structure et de sa voie de synthèse ont permis la recherche d'inhibiteurs et d'antagonistes dont l'utilisation permettra, peut-être, de préciser davantage les mécanismes d'action de ce médiateur. Dans cet article nous nous sommes attachés principalement à résumer les données récentes plaidant en faveur du rôle de ce médiateur dans la physiopathologie bronchopulmonaire.

\section{Biosynthèse} du PAF-acéther

Les mécanismes de libération du PAF-acéther sont complexes : le PAF-acéther est produit à partir des phospholipides membranaires en deux étapes : activation d'une phospholipase $\mathrm{A}_{2}$ qui libère le lyso-PAF, suivie de l'action d'une acétyltransférase qui l'acétyle. L'activité de ces deux enzymes est dépendante de la présence de calcium. De plus, l'acétyltransférase est activée par un processus de phosphorylation probablement catalysé par la protéine kinase C [4]. Le PAF-acéther est hydrolysé par une acétylhydrolase présente dans le plasma et dans divers types cellulaires $[3,5]$.

La phospholipase $\mathrm{A}_{2}$ est également responsable de la libération de l'acide arachidonique, le précurseur des prostaglandines et des leucotriènes. L'interaction entre 


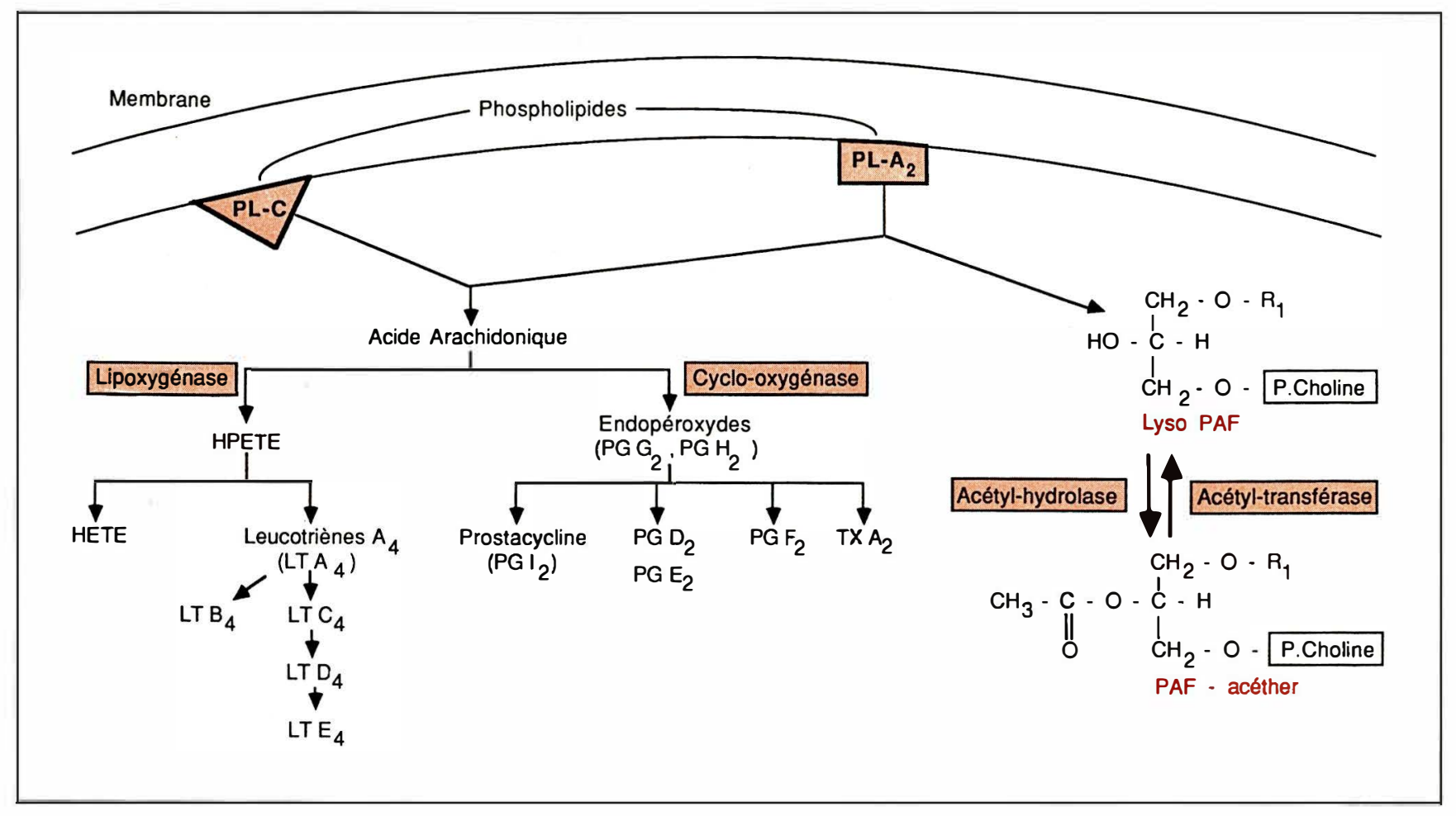

Figure 1. Synthèse de composés lipidiques actifs (PAF-acéther et dérivés de I'acide arachidonique) à partir des phospholipides membranaires. Dans les rectangles et triangle rouges, enzymes impliquées : PL-C = phospholipase $C ; P L-A_{2}=$ phospholipase $A_{2}$. HPETE = acide hydroperoxyéicosatétraénoïque; HETE = acide hydroxyéicosatétraénoïque; $L T=$ leucotriènes ; $P G=$ prostaglandines ; $T X=$ thromboxane. Dans la formule du PAF-acéther: $R_{1}=$ chaîne hydrocarbonée d'un acide gras; $P$-Choline = phosphorylcholine $\left(-P_{-}-O-\left(\mathrm{CH}_{2}\right)_{2}-\mathrm{N}^{+}-\left(\mathrm{CH}_{3}\right)_{3}\right)$. $0-11$

ces différents médiateurs a lieu à divers niveaux, puisque le PAFacéther peut stimuler la libération de métabolites de l'acide arachidonique. De plus, tous ces médiateurs peuvent exercer des actions synergiques, ajoutant ainsi un nouveau degré de complexité à l'étude de la pharmacologie des réactions inflammatoires et allergiques.

\section{Effets biologiques généraux}

Dès la mise à disposition de PAFacéther synthétique [6], différents groupes ont cherché à déterminer ses activités biologiques soit sur divers systèmes cellulaires ou tissulaires isolés soit in vivo. Le PAF-acéther active diverses cellules comme les plaquettes et les polynucléaires neutrophiles (agré$m / s \quad n \circ$ vol. 3 , novembre 87 gation, dégranulation), les éosinophiles (libération de leucotriènes), les macrophages et les monocytes (libération de prostaglandines et d'anions superoxydes) et, comme cela a été démontré plus récemment, les lymphocytes (modulation de la production d'interleukine-2) [3].

Injecté sous la peau, le PAFacéther induit une augmentation de la perméabilité vasculaire accompagnée d'un œdème et de lésions vasculaires. Ces effets sont indépendants des plaquettes chez le cobaye [7], le lapin [8] et le rat [9], bien qu'une accumulation plaquettaire puisse accompagner les modifications de la perméabilité vasculaire. L'injection intradermique de PAF-acéther chez le cobaye provoque une margination et une activation rapides des neutrophiles qui migrent vers le com- partiment extravasculaire [10]. Chez l'homme, la réponse inflammatoire au PAF-acéther est caractérisée par une phase initiale aiguë et par une phase tardive. Administré par voie intradermique, il induit dans un premier temps un œdème localisé et une infiltration des neutrophiles et des monocytes suivis, après environ 24 heures, d'une apparition de lymphocytes. Ces infiltrations cellulaires sont inhibées par un composé à activité anti-allergique, le cromoglycate disodique, injecté à fortes doses en même temps que le PAF-acéther [11].

Des organes comme le cœur et le poumon réagissent au PAFacéther par des modifications comparables à celles observées lors de la stimulation par l'antigène. Ainsi, le PAF-acéther réduit la force de contraction cardiaque et 


\section{RÉFÉRENCES}

1. Benveniste J, Tencé $M$, Varenne $P$, Bidault J, Boullet C, Polonsky J. Semi-synthesis and proposed structure of platelet-activating factor (PAF) : PAF-acether, an alkyl ether analog of lysophosphatidylcholine. CRC Acad Sci 1979 ; 289D : 1037-40.

2. Demopoulos CA, Pinckard RN, Hanahan DJ. Platelet-activating factor. Evidence for 1-0-alkyl-2-acetyl-sn-glyceryl-3-phosphorylcholine as the active component (a new class of lipid chemical mediators). J Biol Chem $1979 ; 254$ : 9355-8

3. Braquet $P$, Touqui L, Shen TY, Vargaftig BB. Perspectives in platelet-activating factor research. Pharmacol Rev (sous presse).

4. Lenihan DJ, Lee TH. Regulation of platelet-activating factor synthesis : modulation of 1-alkyl-2-lyso-sn-glycero-3-phosphocholine acetyl-CoA acetyltransferase by phosphorylation and dephosphorylation in rat spleen microsomes. Biochem Biophys Res Commun 1984; 120 : 834-9.

5. Blank ML, Lee TC, Fitzgerald V, Snyder F. A specific acetyhydrolase for 1-alkyl-2-acetylsn-glycero-3-phosphocholine (a hypotensive and platelet-activating lipid). J Biol Chem 1981; 256 : 175-8.

6. Heymans F, Michel E, Borrel MC, el al. New total synthesis and high solution $1 \mathrm{H}$ NMR spectrum of platelet-activating factor, its enantiomer and racemic mixtures. Biochem Biophys Acla 1981 ; 666 : 230-7.

7. Morley J, Page CP, Paul W. Inflammatory actions of platelet-activating factor (PAFacether) in guinea-pig skin. $\mathrm{Br} J$ Pharmacol $1983 ; 80$ : 503-9.

8. Humphrey DM, Hanahan DJ, Pinckard RN. Induction of leukocytic infiltrates in rabbit skin by acetyl glyceryl ether phosphorylcholine. Lab Invest 1982 ; 47 : 227-34

9. Pirotzky E, Page CP, Roubin R, et al. PAF-acether-induced plasma exudation in rat skin is independent of platelet and neutrophils. Microcirculation Endothelium Lymphatics 1984; 1 : 107-22.

10. Bourgain RH, Andries R, Maes L, Sèdivy $P$, Braquet $P$. Proceedings of the First Sandoz Research Symposium. In : Winslow CM, Lee ML, eds. New Horizons in Platelet-activating Factor Research. London : J. Wiley, 1987 (sous presse).

11. Basran GS, Page CP, Paul W, Morley J. Cromoglycate (DSCG) inhibits responses to platelet-activating factor (PAF-acether) in man : an alternative mode of action for DSCG in asthma ? Eur $J$ Pharmacol $1983 ; 86: 143-4$.

12. Benveniste J, Boullet C, Brink C, Labat C. The actions of PAF-acether (plateletactivating factor) on guinea-pig isolated heart le débit coronarien chez le cobaye [12]. Ces effets, accompagnés d'une libération de thromboxane A2 (figure 1) et d'histamine, sont observes également lors du choc anaphylactique. Ces libérations de médiateurs, induites par le PAF-acéther et par l'antigène, sont inhibées par les antagonistes spécifiques du PAFacéther [13], ce qui plaide en faveur d'un rôle de ce médiateur dans les réactions d'hypersensibilité immédiate au niveau du cœur. Des travaux récents [14] ont montré que les effets cardiaques du PAF-acéther étaient liés à l'activation de la voie de la lipoxygénase puisque le composé FPL 55712, un antagoniste des récepteurs pour les leucotriènes (figure 1), inhibe fortement l'action du médiateur. Cette activation de la voie de la lipoxygénase par le $\mathrm{PAF}$-acéther n'est pas spécifique du tissu cardiaque puisqu'elle est également observée dans le tissu pulmonaire (voir plus loin). Lors du choc anaphylactique, divers médiateurs comme le PAFacéther [15], le thromboxane A2 et l'histamine [13] sont libérés par le cœur isolé de cobaye. En conséquence, la contribution respective de ces agents dans les modifications des paramètres cardiaques reste à déterminer.

\section{Les réactions allergiques et inflammatoires}

- Effet bronchoconstricteur. De nombreuses études ont impliqué le PAF-acéther dans les réactions allergiques bronchopulmonaires. Les premiers résultats ont été obtenus chez le cobaye anesthésié où le PAF-acéther, injecté par voie intraveineuse à des doses de l'ordre de quelques nanogrammes, induit une bronchoconstriction accompagnée d'une hypotension et d'une chute du nombre des plaquettes et des leucocytes circulants [16]. Il est intéressant de noter que, lorsque le PAF-acéther est perfusé par voie intraveineuse à des doses de 1 à $44 \mathrm{ng} / \mathrm{kg} / \mathrm{min}$, la leucopénie et l'hypotension apparaissent à la plus faible dose du médiateur, tandis que la bron- choconstriction et la thrombopénie nécessitent des doses plus élevées. Le fait que la bronchoconstriction induite par le PAF-acéther ne soit pas due à la formation de prostaglandines a été démontré par l'absence d'effet antagoniste de l'aspirine, un inhibiteur connu de la cyclooxygénase [16]. En revanche, la déplétion en plaquettes, ou l'utilisation de prostacycline, un inhibiteur de leur activation, suppriment la réponse bronchoconstrictrice au PAF-acéther, indiquant que cette dernière est liée à la sécrétion par ces éléments de médiateurs autres que les métabolites de la voie de la cyclooxygénase $[16,17]$.

- Activation plaquettaire par le PAF-acéther in vivo. La thrombopénie successive à l'administration intraveineuse de PAF-acéther pourrait être liée à une agrégation intravasculaire des plaquettes suivie d'une séquestration des agrégats dans la microcirculation. L'utilisation d'une méthode de marquage des plaquettes par un traceur radioactif a permis de démontrer, à la suite de l'injection de PAF-acéther, une accumulation très rapide de ces éléments dans le tissu pulmonaire [18]. Des études morphologiques ont également montré que le PAF-acéther, de même que l'antigène, induit chez le cobaye le recrutement des plaquettes qui migrent en direction du compartiment extravasculaire, à proximité des muscles lisses bronchiques [19]. L'effet du PAF-acéther sur le recrutement cellulaire ne se limite pas aux poumons, puisqu'il a lieu également lorsque des artérioles mésentériques de cobaye sont exposées au médiateur (figure 2).

Bien que la contribution précise des plaquettes pour le déclenchement ou la persistance de l'hyperréactivité bronchique ne soit pas encore élucidée, leur activation pourrait être importante dans les modifications des fonctions bronchopulmonaires observées dans l'asthme.

Cette activation, qui est observée chez les sujets asthmatiques pendant les tests de provocation [20], serait accompagnée d'une libéra- 


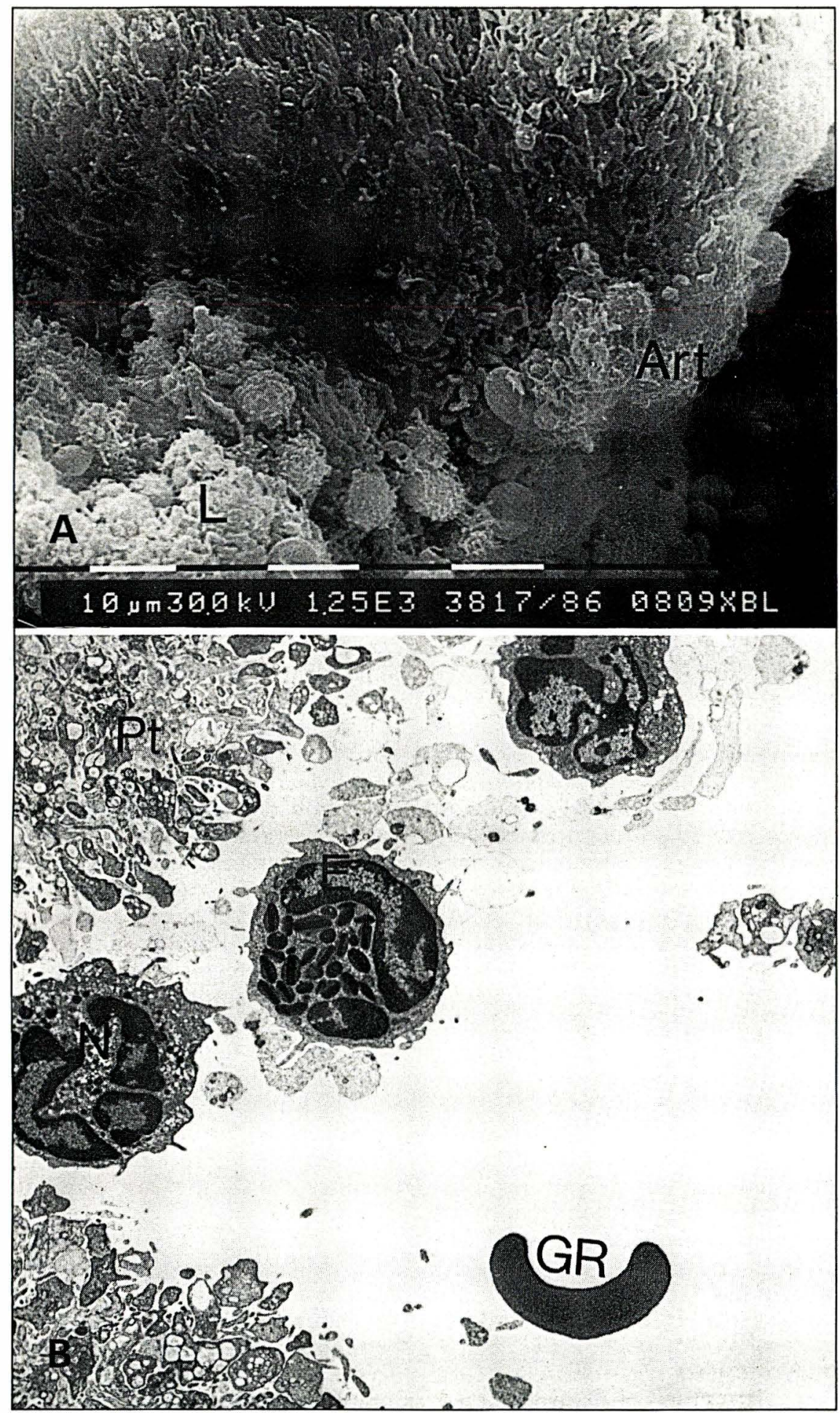

Figure 2. Microscopie électronique d'une artériole mésentérique de cobaye traitée in situ par $1 \mu$ M de PAF-acéther. A. Par balayage : 15 minutes après, on note un amas de plaquettes $(P t)$ adhérentes à l'endothélium et agrégées, la paroi artérielle (Art) à proximité d'un érythrocyte (GR), et un amas de leucocytes ( $L$ ) en train $d^{\prime}$ envahir le thrombus. B. Par transmission : diverses cellules inflammatoires ( $N=$ neutrophiles ; $E=$ éosinophiles), ainsi qu'un érythrocyte (GR) et des plaquettes (Pt) partiellement dégranulées. (Ces deux photographies nous ont été gracieusement fournies par le $\mathrm{Pr}$ R.H. Bourgain et le $\mathrm{Dr}$ R. Andries, Laboratorium voor Fysiologie, Vree Universitet, Bruxelles, Belgique). tion de PAF-acéther [3].

- Rôle du PAF-acéther dans les réactions d'hypersensibilité immédiate. De nombreux résultats expérimentaux plaident en faveur d'un rôle du PAF-acéther dans l'hypersensibilité immédiate, bien que les effets bronchopulmonaires de ce médiateur ne puissent être considérés comme seuls responsables de l'asthme bronchique. De plus, le PAF-acéther est capable de reproduire chez l'homme et chez l'animal la réaction tardive de l'asthme, caractérisée par une hyper-réactivité bronchique et une migration de cellules inflammatoires, notamment d'éosinophiles, dans le poumon $[21,22]$. Il a également été montré que le PAFacéther, administré par aérosol au cobaye, entraîne une bronchoconstriction indépendante de l'activation plaquettaire [23] et susceptible de désensibilisation lorsque la stimulation avec ce médiateur est répétée. En revanche, les animaux exposés à un aérosol de PAFacéther, continuent à répondre par une bronchoconstriction lors de son injection intraveineuse [24]. Ceci suggère que, selon la voie d'administration choisie, le PAF-acéther induit une activation préférentielle de certains types cellulaires. En effet, il est probable que les cellules alvéolaires, notamment les macrophages, sont stimulées lors de l'administration du médiateur par aérosol. $\mathrm{Au}$ contraire, les plaquettes circulantes sont la cible de l'autacoïde* après son injection par voie intraveineuse.

Dans les deux cas, une bronchoconstriction se développe, bien que sa modulation pharmacologique soit très différente. Pour ce qui concerne les macrophages alvéolaires, différentes études ont montré qu'ils pourraient constituer une cible pulmonaire pour le PAF-acéther. En effet, ces cellules sont activées par le PAFacéther [23] et, lorsqu'ils proviennent de sujets allergiques, leur stimulation in vitro avec l'antigène spécifique est accompagnée d'une

- Aulacoïde : médialeur de l'inflammation. 


\section{RÉFÉRENCES}

13. Berti F, Rossoni G. PAF, Ginkgolides and active anaphylactic shock in lung and heart. In : Braquet P, ed. The Ginkgolides : Chemistry, Biology, Pharmacology and Clinical Perspectives. Methods and Findings. Barcelona : JR. Prous, 1987 (sous presse).

14. Piper PJ, Stewart AG. Antagonism of vasoconstriction induced by platelet-activating factor in guinea-pig perfused hearts by selective platelet-activating factor receptor antagonist. Br J Pharmacol 1987 ; 90 : 771-83.

15. Levi R, Burke JA, Guo ZG, el al. Acetyl glyceryl ether phosphorylcholine (AGEPC). A putative mediator of cardiac anaphylaxis in the guinea-pig. Circ Res 1984 ; 54 : 117-24.

16. Vargaftig BB, Lefort J, Chignard M, Benveniste $J$. Platelet-activating factor induces a platelet-dependent bronchoconstriction unrelated to the formation of prostaglandin derivatives. Eur J Pharmacol 1980 ; 65 : 185-92.

17. Chignard M, Wal F, Lefort J, Vargaftig $B B$. Inhibition by sulphinpyrazone of the platelet-dependent bronchoconstriction due to platelet-activating factor (PAF-acether) in the guinea-pig. Eur J Pharmacol 1982 ; 78 : 71-9.

18. Page CP, Paul W, Morley J. An in vivo model for studying platelet aggregation and disaggregation. Thromb Haemost $1983 ; 47$ : 210-3.

19. Lellouch-Tubiana A, Lefort J, Pirotzky E, Vargaftig BB, Pfister A. Ultrastructural evidence for extravascular platelet recruitment in the lung upon intravenous injection of plateletactivating factor (PAF-acether) to guinea-pigs. Br J Exp Pathol 1985 ; 66 : 345-55.

20. Capron A, Ameisen JC, Joseph M, Auriault C, Tonnel AB, Caen J. New functions for platelets and their pathological implications. Int Arch Allergy Appl Immunol 1985 ; $77: 107-14$

21. Cuss FM, Dixon CM, Barnes PJ. Inhaled platelet-activating factor causes bronchoconstriction and increased bronchial reactivity in man. Am Rev Respir Dis 1986 ; 133 : A 212.

22. Lellouch-Tubiana A, Lefort J, Pfister A, Vargaftig BB. Interactions between granulocytes and platelets with the guinea-pig lungs in passive anaphylactic shock. Correlations with PAF-acether-induced lesions. Int Arch Allergy Appl Immunol 1987 (sous presse).

23. Maridonneau-Parini I, Lagente V, Lefort J, Randon J, Russo-Marie F, Vargaftig BB. Desensitization to PAF-induced bronchoconstriction and to activation of alveolar macrophages by repeated inhalations of PAF in the guinea-pig. Biochem Biophys Res Commun 1985 ; $131: 42-9$. libération dans le surnageant de lyso-PAF et de PAF-acéther [25]. Un rôle pour le PAF-acéther dans les réactions allergiques a été également proposé sur la base des résultats montrant la formation de ce médiateur au cours du choc anaphylactique systémique chez le lapin [3]. De plus, une libération de lyso-PAF, précurseur et catabolite du PAF-acéther, a été mesurée à partir de poumons provenant de cobayes sensibilisés activement et stimulés par l'antigène $[26,27]$. Néanmoins, comme la quasi-totalité des antagonistes du PAF-acéther sont inactifs visà-vis du choc anaphylactique actif systémique chez l'animal, les mécanismes à travers lesquels ce médiateur participe au phénomène restent identifier.

- Effet activateur du PAFacéther sur les éosinophiles. De nombreux travaux, passés et récents, ont souligné l'importance potentielle des éosinophiles dans le phénomène asthmatique. Ceci est caractéristique de la réaction tardive chez l'homme où l'on observe une infiltration massive de neutrophiles et d'éosinophiles dans le parenchyme pulmonaire et les voies aériennes [28]. Le PAFacéther est connu comme étant un activateur des éosinophiles dans différents modèles. Ainsi, il active le chimiotactisme des éosinophiles humains [29], probablement par l'interaction avec un site de liaison spécifique, puisque les antagonistes spécifiques du PAFacéther inhibent la capacité locomotrice de ces cellules [3]. De plus, il a été démontré que des injections intracutanées de PAFacéther induisent, chez les sujets allergiques exclusivement, une importante infiltration d'éosinophiles, accompagnée d'une dégranulation [30]. En revanche, chez les sujets sains, le neutrophile est le seul type cellulaire dont la migration soit activée lors d'une stimulation par le PAF-acéther. L'intensité de la réponse des éosinophiles chez les allergiques est indépendante de leur taux dans la circulation et du niveau plasmatique d'IgE [30]. Ces résultats suggèrent que les éosinophiles sont recrutés par le PAF-acéther de façon préférentielle chez les allergiques et que leur réactivité semble qualitativement différente de celle des sujets sains. Un autre argument direct en faveur de l'implication du PAF-acéther dans les réactions d'hypersensibilité est la libération accrue de leucotriène $\mathrm{C}_{4}$, induite après traitement par le PAF-acéther d'éosinophiles de sujets asthmatiques [31]. Cet effet est inhibé par le composé BN 52021, antagoniste du PAFacéther, suggérant là encore la présence d'un récepteur spécifique.

D'autre part, chez le cobaye, une heure après l'inhalation de PAFacéther, de nombreux agrégats de polynucléaires neutrophiles sont observés par microscopie électronique dans les capillaires alvéolaires (figure $3 A$ ). Ces phénomènes, ainsi qu'une accumulation d'éosinophiles et de macrophages dans la muqueuse sub-bronchique, sont observés également après un choc anaphylactique induit par l'administration de l'antigène par aérosol [22] (figure 3B). La similitude des phénomènes observés après stimulation soit avec l'antigène, soit avec le PAF-acéther, suggère que ce dérivé lipidique joue bien un rôle déterminant dans la pathologie asthmatique.

- Effet sur le tissu pulmonaire isolé. Injecté dans l'artère pulmonaire de poumons isolés et perfusés de cobayes, le PAF-acéther induit une bronchoconstriction associée à la libération de thromboxane A2 [32]. Ces résultats démontrent que le tissu pulmonaire est une cible directe du PAF-acéther puisque ces expériences ont été réalisées en l'absence de cellules circulantes. Cet effet bronchoconstricteur du PAFacéther est dans ce cas largement inhibé par l'aspirine, suggérant la contribution de la vole de la cyclooxygénase. Les travaux de Voelkel et al. [33] ont montré que l'administration de PAF-acéther à des poumons perfusés de rats induit la libération de leucotriènes C4 et D4 (figure 1), associée au développement d'un œdème. En effet, l'adjonction de diéthylcarba- 


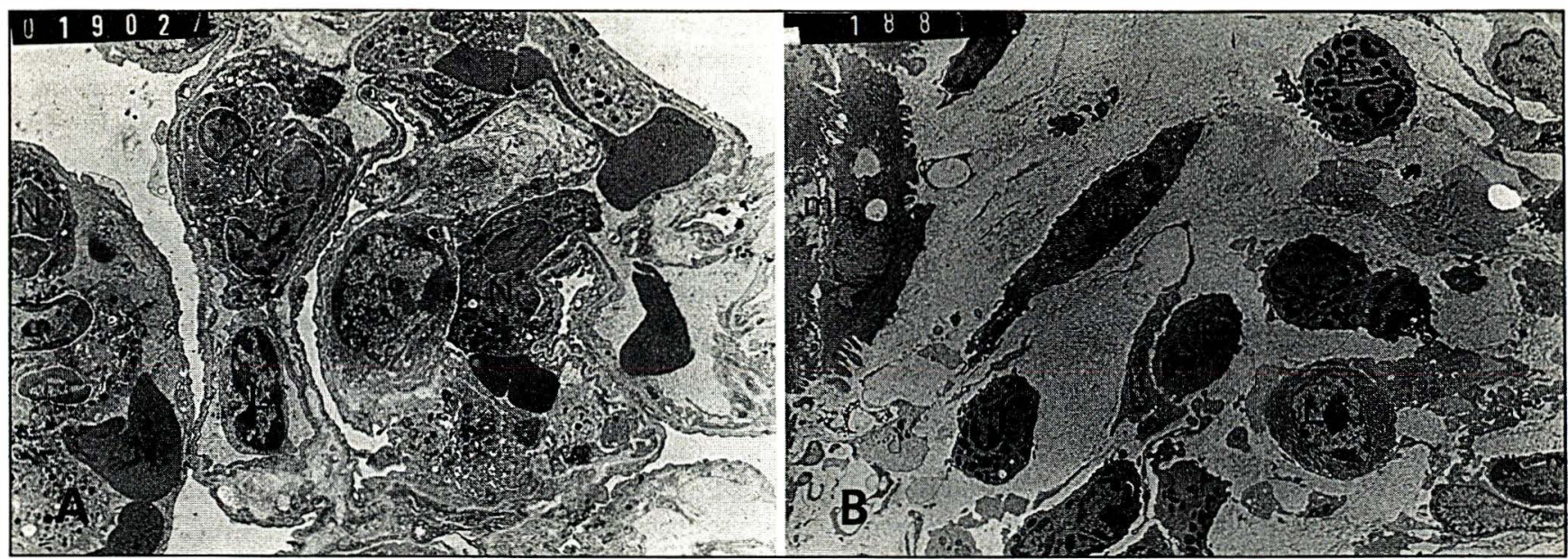

Figure 3. Microscopie électronique par transmission du parenchyme pulmonaire de cobaye. A. Une heure après stimulation par un aérosol de PAF-acéther $(330 \mu \mathrm{g} / \mathrm{ml}$ pendant 2 minutes). On observe une accumulation importante de neutrophiles $(N)$ et quelques lymphocytes $(L)$ dans les capillaires alvéolaires. B. Provenant d'un cobaye sensibilisé passivement 1 heure après l'aérosol de l'antigène ovalbumine $(10 \mathrm{mg} / \mathrm{ml}$ pendant 2 minutes). L'image montre la présence d'éosinophiles (E), de lymphocytes (L) et de mastocytes muqueux (Mm) dans l'épithelium et à proximité du muscle lisse bronchique (mb). (Clichés gracieusement fournis par le Dr A. Lellouch-Tubiana, laboratoire d'histologie, faculté de médecine Necker-Enfants Malades, Paris).

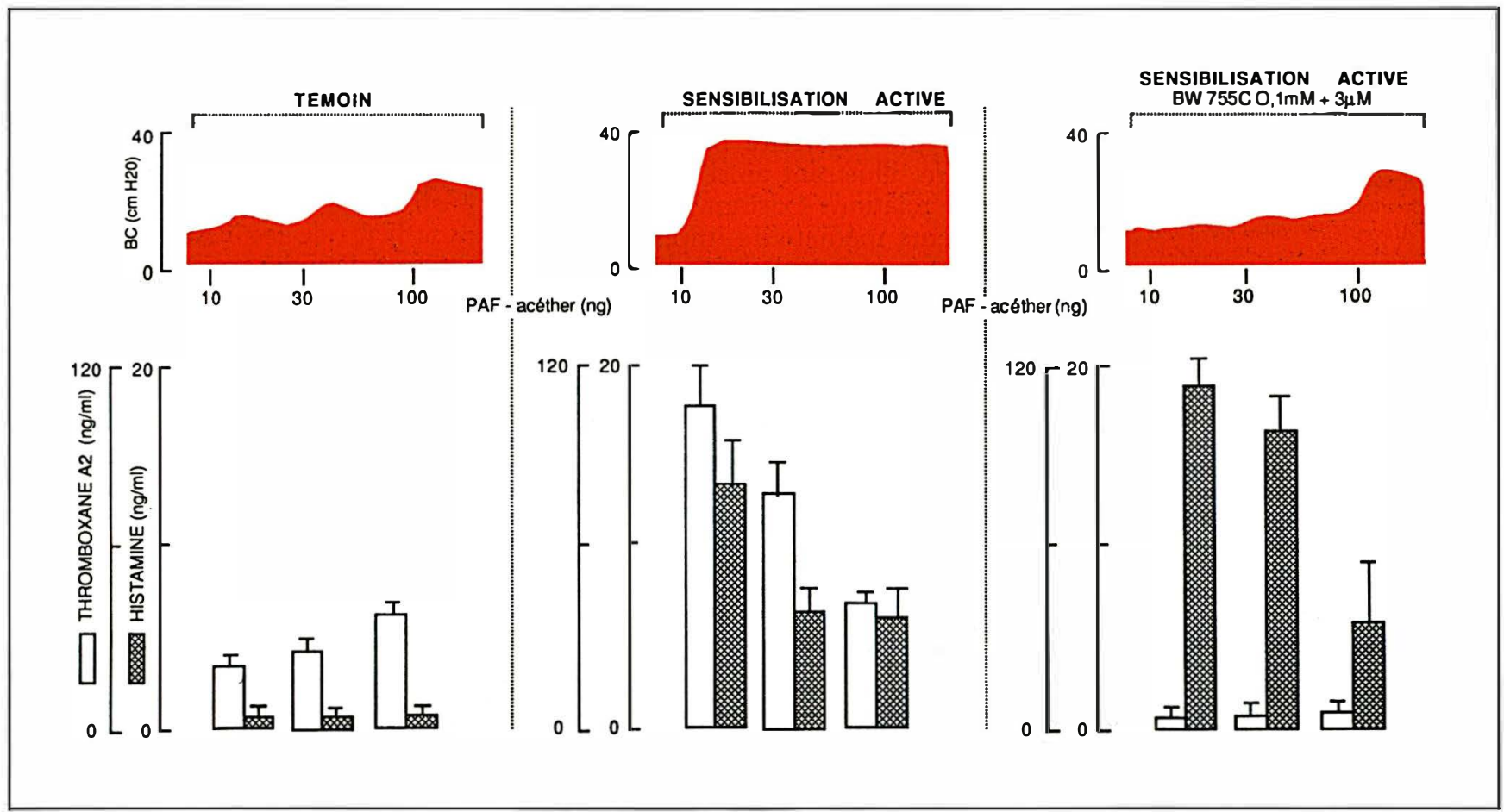

Figure 4. Bronchoconstriction (partie supérieure) et libération de thromboxane (TX)A2 et d'histamine (partie inférieure) induites par l'administration de doses successives $(10,30,100 \mathrm{ng})$ de PAF-acéther à des poumons perfusés provenant de cobayes contrôlés (gauche), ou sensibilisés activement à l'ovalbumine (centre et droite). Les résultats présentés sur le panneau de droite sont ceux obtenus en présence de $0,1 \mathrm{mM}$ de $\mathrm{BW} 755 \mathrm{C}$ (un inhibiteur de la cyclooxygénase et de la lipoxygénase) et de $0,3 \mu \mathrm{M}$ de mépyramine (un anti-histaminique).

$m / s$ n 9 vol. 3, nooembre 87 


\section{RÉFÉRENCES}

24. Cirino M, Lagente V, Lefort J, Vargaftig BB. Studies with BN 52021 demonstrated the involvement of PAF-acether in IgEdependent anaphylactic bronchoconstriction. Proslaglandins $1986 ; 32$ : 121-6.

25. Arnoux B, Simoes-Caeiro MH, Landes A, Mathieu M, Duroux P, Benveniste J. Alveolar macrophages from asthmatic patients release platelet-activating factor (PAF-acether) and lyso PAF-acether when stimulated with specific allergen. Am Rev Respir Dis 1982; 125: 70 (abstract).

26. Rotilio D, Lefort J, Detsouli A, Vargaftig BB. Absence de contribution du PAFacéther à la réponse anaphylactique pulmonaire in vitro chez le cobaye. J Pharmacol $1983 ; 14$ (suppl 1) : 97-109.

27. Fitzgerald MF, Moncada S, Parente L. The anaphylactic release of platelet-activating factor from perfused guinea-pig lungs. $B r J$ Pharmecol 1986 ; 88 : 149-53.

28. De Monchy JG, Kauffman HF, Venge $P$, et al. Bronchoalveolar eosinophilia during allergen-induced late asthmatic reactions. $A m$ Rev Respir $D$ is 1985 ; 131 : 373-6.

29. Wardlaw AJ, Kay AB. PAF-acether is a potent chemotactic factor for human eosinophils. J Allergy Clin Immunol 1986; $77: 236$.

30. Henocq E, Vargaftig BB. Accumulation of eosinophils in response to intracutaneous PAFacether and allergens in man. Lancet 1986 ; i : 1378-9.

31. Borgeat P, Fruteau de Laclos B, Rabinovich $\mathrm{H}$, et al. Eosinophils-rich human polymorphonuclear leukocyte preparations characteristically release leukotriene $\mathrm{C} 4$ on ionophore A23187 challenge. J Allergy Clin Immunol 1984 ; 74 : 310-5.

32. Lefort J, Rotilio D, Vargaftig BB. The platelet-independent release of thromboxane A2 by $\mathrm{PAF}$-acether from guinea-pig lungs involves mechanism distinct from those of leukotrienes. Br J Pharmacol 1984; 82 : 565-75.

33. Voelkel NF, Worthen S, Reeves JT, Henson PM, Murphy RC. Non immunological production of leukotrienes induced by plateletactivating factor. Science $1982 ; 218$ : 286-8.

34. Lefort J, Malanchère E, Pretolani M, Vargaftig BB. Immunisation induces bronchial hyper-reactivity and increased mediator release from guinea-pig lungs. Br $J$ Pharmacol 1986 ; mazine, un inhibiteur de la synthèse des leucotriènes, inhibe les effets du PAF-acéther. L'ensemble de ces résultats suggère qu'en dehors de ses effets propres, le PAF-acéther peut agir à travers la production de médiateurs secondaires comme les prostaglandines et les leucotriènes.

- État immunitaire et réponse du tissu pulmonaire. Des travaux récents de notre laboratoire ont montré que la réactivité au PAF-acéther du tissu pulmonaire de cobayes sensibilisés activement est exacerbée [34], le médiateur induisant dans ce cas une bronchoconstriction accrue et une libération de thromboxane A2 plus importante que lors du traitement $\mathrm{du}$ tissu normal. De plus, seuls des poumons provenant d'animaux sensibilisés et non ceux d'animaux normaux appariés sécrètent alors de l'histamine (figure 4, p. 513). Ces résultats indiquent que l'état immunitaire altère quantitativement et qualitativement la réponse du poumon à la stimulation par le PAF-acéther. Des expériences préliminaires montrent que les dérivés de la lipoxygénase participent de façon déterminante au phénomène d'hyper-réactivité bronchopulmonaire, illustrant ainsi la complexité des relations existant entre les différents médiateurs impliqués dans les réactions allergiques.

\section{Conclusions}

Sur la base de ces données, il est évident que le traitement de la crise asthmatique ne saurait trouver sa solution dans l'emploi d'un seul type d'inhibiteur spécifique. Cependant, l'utilisation d'antagonistes sélectifs des récepteurs des divers médiateurs impliqués, dont ceux du PAF-acéther, devrait permettre de déterminer l'éventuelle contribution de ces médiateurs dans l'installation du " terrain atopique ". Pour la compréhension de ce dernier phénomène, l'étude des modifications de la sensibilité du système bronchopulmonaire en relation avec les infiltrats cellulaires et l'état immunitaire présente un intérêt tout particulier

\section{Summary}

PAF-acether I (platelet-activating factor) is a potent lipid mediator originally shown to be released from antigenstimulated rabbit basophils. Other inflammatory cells, i.e. neutrophils, macrophages, eosinophils and platelets, also synthetize and/or respond to PAF-acether when stimulated in vitro. PAF-acether is also a potent in vivo agonist which triggers several pathological changes in lung and heart functions. It increases the vascular permeability, induces edema and cellular infiltration, particularly of eosinophils into the lung. Because of its capacity to evoke the release of secondary mediators, such as prostaglandins and leukotrienes from different cell types and organs, a role for PAF-acether as an enhancer of the inflammatory response has been hypothetized. Recently, PAFacether has been shown to induce a long-lasting, nonspecific increase in bronchial responsiveness in animal and man. This property may be relevant in the pathogenesis of asthma and further strengthens the key role for PAF-acether in the development of allergic reactions.

\section{TIRÉS À PART}

M. Pretolani, B. Vargaftig : unité de pharmacologie cellulaire, unité associée Institut Pasteur/Inserm, U. 285, 25, rue du DocteurRoux, 75015 Paris. 